\title{
Recuperação de Imagens baseadas na textura
}

Yamashita, B. H. M.

Escola Politécnica de Pernambuco

Universidade de Pernambuco

50.720-001 - Recife, Brasil

bhmy@ecomp.poli.br
Fernandes, B. J. T.

Escola Politécnica de Pernambuco

Universidade de Pernambuco

50.720-001 - Recife, Brasil

Resumo O reconhecimento de características que descrevem uma imagem de maneira concisa e eficiente é uma tarefa relativamente complexa para ser executada. O objetivo deste trabalho é demonstrar como a descrição pode ser feita a através da textura de imagens. Será mostrado como os descritores de textura funcionam e com um estudo comparativo entre seus resultados foi realizado a fim de obter a melhor descrição.

\footnotetext{
Abs-

The recognition of features that describe an image concisely and efficiently is a relatively complex tract task to be performed. The objective of this study is to demonstrate how an image description can be obtained through the texture images. It will be demonstrated how the texture descriptors operate and a comparative study between the results was carried out to obtain the best description.
} 


\section{Introdução}

Analisar imagens em meio digital é de fundamental importância para diversas áreas de conhecimento. O Processamento Digital de Imagens (Gonzalez [11]) é uma área de pesquisa responsável por tal função. Utilizado amplamente na medicina, artes, biologia, automação industrial, microscopia, dentre outros, é uma área que vem crescendo bastante no ramo de pesquisas, cujo seu principal objetivo é obter uma caracterização que contenha informação suficiente para distinguir regiões, objetos ou texturas de interesse, de forma confiável e com o mínimo de intervenção humana (Pedrini [15]).

Diversos trabalhos podem ser citados relacionando o estudo de análise de imagens com as muitas áreas de sua aplicação. Na medicina, por exemplo, Petrakis [16] utilizou grafos para representar as relações espaciais entre objetos voltados para área de saúde. No reconhecimento de escritas por regiões Smith [7] realizou um trabalho sobre a análise de imagens para detecção de caracteres (strings). Reaes [9] mostrou como pode ser feito Reconhecimento de faces em fotos $3 \times 4$, onde são comparadas duas técnicas de Segmentação, uma baseada em bordas e outra baseada na cor da pele. No campo da arte, o principal software utilizado para manipulações de imagens o Adobe Photoshop, explorado por Thomson [19], é bastante utilizado para área de design e possui diversos algoritmos com base em algoritmos de Processamento de Imagens já programados. Haralick [12] e Abbadeni [1] propuseram descrições de textura, através de cálculos matemáticos, tornando possível obter as características de uma imagem através de descritores. Em Haralick [12][13] é realizado um trabalho explorando como tema o computador e a visão de robôs, onde são propostos técnicas de visão computacional. Em Asdanlogan [4] a Recuperação de Imagens é utilizada para busca de vídeos (que é um conjunto de imagens seqüenciadas).

É possível observar que as aplicações para Processamento de Imagens é bastante ampla, extensa e permite a resolução de diversos problemas que envolvam imagens e ainda pouco exploradas. Novas e antigas pesquisas de temas relevantes ainda podem ser feitas e melhoradas em cima da área de Processamento de Imagens.

A internet, por exemplo, é um dos meios de comunicação e de transmissão de dados em que o Processamento de Imagens é bastante aplicado. CBIR (Content-Based Image Retrieval, ou Recuperação de Imagem baseada no conteúdo), por exemplo, é muito utilizada em buscas por imagens em sites como Picasa (Google) e Flickr (Yahoo). Tema que foi amplamente discutido em Datta [10] e será o foco desta pesquisa para Recuperação de Imagens de Textura.
Neste trabalho, foi realizada uma comparação dentre os descritores propostos por Haralick [12], baseado na matriz de co-ocorrência, e Abbadeni [1], baseado na função de autocorrelação. Vários testes foram realizados a fim de encontrar uma melhor descrição (com maior taxa de acerto) para a Recuperação de Imagem baseada na textura.

\section{Metodologia utilizada}

As ferramentas de estudo utilizadas para a realização dos testes foram o programa MatLab (mathworks.com) e Eclipse (eclipse.org), este segundo tendo como suporte para Linguagem Java (oracle.com). Ambos possuem uma ampla biblioteca para estudo em Processamento Digital de Imagens e permitiram realizar o estudo prático para obtenção dos resultados neste trabalho.

Como ferramenta de pesquisa, os sites do IEEE (ieee.org) e ACM (acm.org), sites acadêmicos com artigos renomados de diversas áreas em tecnologia, foram utilizados juntamente com os livros de Gonzalez [11] e Pedrini [15].

Nos testes, foi utilizado o banco de dados de Brodatz [6], onde é possível encontrar várias imagens ricas em texturas, que serviram como base para este estudo de Recuperação de Imagens. Apesar de ser antigo, ele é utilizado por muitos pesquisadores na área de Processamento de Imagens. Na fig. 1 alguns exemplos de imagens do banco.

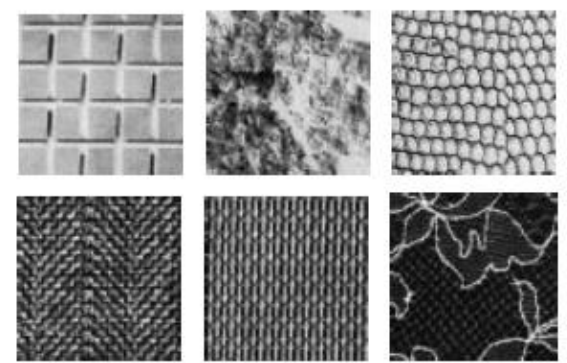

Fig. 1. Exemplos do Banco de Brodatz(1966).

Para realizar o cálculo das similaridades obtidas nos resultados foi utilizada a curva de recall, que é sempre utilizada nas pesquisas de Recuperação de Imagens para verificar os resultados.

\section{Recuperação de Imagem baseada no Conteúdo (CBIR)}

A internet tornou-se a principal ferramenta de busca utilizada para buscas. Em Datta [10], uma análise é feita mensurando todos os tipos de usuários e suas pesquisas 
relacionadas. Foi observado que a grande maioria das buscas pode ser realizada por base em palavras-chave, quando se trata de textos. A grande dificuldade está quando esta busca depende do reconhecimento de imagens, que é onde a CBIR (Recuperação de Imagens Baseada no Conteúdo) é empregada. Onde é utilizada? Como funciona?

Seus os métodos para reconhecimento de padrões não são nada triviais (Datta [10]) tanto em termos de algoritmos e de utilização, pois tratam com operações de matrizes. A idéia principal de um sistema de Recuperação de Imagens por Imagem é de que, dada uma imagem, o sistema deverá retornar outras com texturas similares. Em outras palavras, se uma imagem possui uma textura de madeira, outras, com esta mesma textura deverão ser retornadas do banco de dados. Em Aigrain [3], Rui [17], Smeulders [18] e Snoek [8] a Recuperação de Imagens é bem analisada e discutida quanto ao estado da arte e suas aplicações. Porém como calcular esta similaridade?

Existe uma propriedade da imagem, denominada de textura. Segundo Haralick [12] uma imagem de textura é descrita pelo número de tipos de suas primitivas com uma organização espacial, ou seja, uma informação visual que permite a identificação de uma dada imagem.

Em Gonzalez [11] e Pedrini [15] a textura é tratada com diversas análises. Na Recuperação de Imagens, essas análises da textura tornam possível a realização de cálculos matemáticos para, com valores numéricos, descrever uma imagem. Diversas características podem ser exploradas em Haralick [12] e Abbadeni [1], nas quais são propostos diversos descritores responsáveis pela obtenção dessas características presentes na textura. A função destes descritores é de descrever as texturas permitindo que, depois de processadas as características (contraste, homogeneidade, entre outras) é possível realizar cálculos matemáticos para encontrar a similaridade entre as imagens.

\section{Análise de Texturas}

Definido o processo da metodologia utilizada para a curva de recall os detalhamentos sobre o mesmo poderão ser analisados. A análise de Textura é tratada em Pedrini [15] e Gonzalez [11] relacionando vários cálculos com bases estatísticas a fim de obter valores das características.

Porém, antes de descrever como funcionam os descritores de textura, é preciso demonstrar como funcionam o filtro de Gabor e os Descritores de Textura de Haralick [12] e Abbadeni [1], mencionado no processo para obtenção dos resultados.

\subsection{Filtro de Gabor}

Explorado por Afshang [2], o filtro de Gabor é uma técnica que pode ser utilizada para melhorar o resultado obtido na curva de recall (Zhang [20]). Sua aplicação diminui consideravelmente o ruído na imagem dependendo dos parâmetros nele utilizados.

Matematicamente, para utilização de qualquer filtro em uma imagem, basta que, depois de calculado sua máscara, uma operação de convolução seja realizada entre o sinal da imagem com o sinal do filtro. Feito este processo, uma nova imagem filtrada é retornada. Na equação 1, a fórmula do Filtro de Gabor.

$$
G(x, y ; f, \theta)=\exp \left[\frac{1}{2}\left(\frac{x^{2}}{\sigma_{x}^{2}}+\frac{y^{2}}{\sigma_{y}^{2}}\right)\right] \cdot \exp (2 \pi \cdot j \cdot f \cdot x)
$$

Para facilitar sua programação, a regra de Euler deve ser aplicada, como mostrado na equação 2 .

$$
\exp (2 \pi \cdot j \cdot f \cdot x)=\cos (2 \pi \cdot j \cdot f \cdot x)+j \cdot \sin (2 \pi \cdot j \cdot f \cdot x)
$$

Este filtro é composto a partir do produto entre uma função gaussiana e uma função harmônica (sinusóide). Nela, temos quatro parâmetros: $\sigma_{x}^{2} \mathrm{e}: \sigma_{y}^{2}$ são os desvios padrões que vêm da equação gaussiana, $f$ é a freqüência da função harmônica definida sobre a função de gabor e $\theta$ representa a indicação, e nele que se define se o filtro terá um bom resultado ou não, como demonstrado nas fig. 2 .

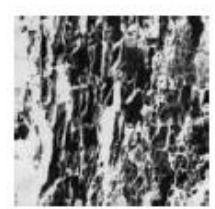

(a)

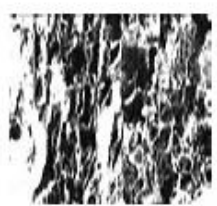

(b)



(c)
Fig 2. (a) Imagem Original. (b) e (c) Imagens Filtradas com o Filtro de Gabor.

Na fig. 2.b o filtro foi utilizado com os parâmetros corretos. Por outro lado a fig. 2.c teve uma utilização errônea do filtro. Como é possível notar, o primeiro resultado foi muito mais satisfatório que o segundo. A diferença entre eles deve-se a orientação escolhida para cada caso, $\theta=$ $\pi / 10$ e $\theta=\pi / 2$ respectivamente. Tais números dados aos parâmetros foram puramente experimentais, já que para algumas imagens essa variação na indicação pode mudar de imagem para imagem, assim como os desvios e freqüências. Para os resultados desta pesquisa, foram utilizados os parâmetros: desvios padrões foram fixados em 
4 e 20 para x e y respectivamente, freqüência em 20 e indicação em (Afshang, [2]).

\subsection{Descritores de Textura Baseados na Ma- trix de Co-ocorência}

Após o estudo sobre o filtro de Gabor, o foco deste trabalho prossegue para seu problema central: a descrição de texturas. Iniciando pelos descritores baseados na matriz de co-ocorrência, propostos por Haralick [12]. Apesar de serem antigos, seus descritores são utilizados como referência em muitas pesquisas recentes que visam à descrição da textura (Abbadeni [1]). Foram propostas mais de 14 propriedades de características, porém Baraldi [5] mostrou que apenas seis eram realmente relevantes. Desses seis, cinco descritores foram programados: Máxima Probabilidade, Contraste, Homogeneidade, Uniformidade e Correlação.

Para poder calculá-los, é necessário obter a matriz de co-ocorrência $p(i, j)$. Ela é obtida através da freqüência entre tons vizinhos. $\mathrm{O}$ armazenamento dessas freqüências é feito como mostrado na fig. 3 .

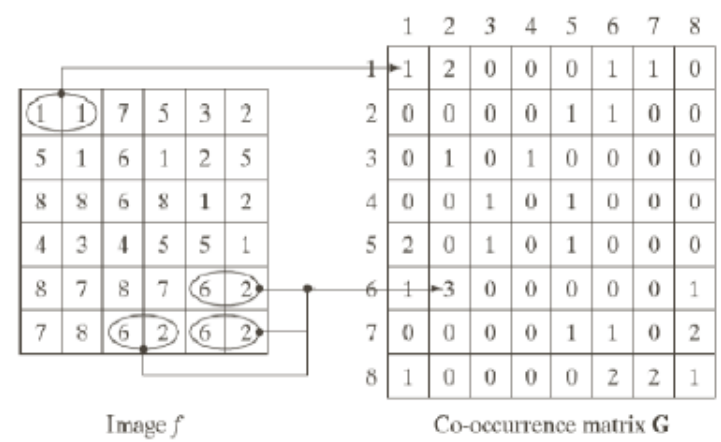

Fig 3. Imagem ilustrativa do cálculo da matriz de Coocorrência G, retirada de Gonzalez (2010).

Calculada a matriz $\mathrm{G}$, é necessário realizar sua normalização, dada como mostrado na equação 3 .

$$
p(i, j)=\frac{g(i, j)}{n}
$$

Onde é a soma das frequências entre os tons de cinza. Depois de calculada a Matriz de co-ocorrência da imagem, a descrição pode ser calculada. Na página seguinte, uma síntese dos descritores estudados, propostos por Haralick [12], com suas respectivas equações:
1. Máxima Probabilidade: Medida mais simples proposta por Haralick [12], consta na observação do maior valor contido na matriz de co-ocorrência e é dada pela equação 4.

$$
f_{\text {maxProb }}=\max _{\mathrm{i}, \mathrm{j}}(p(i, j))
$$

2. Contraste: Quando uma imagem apresenta alta freqüência entre tons de cinza altos e baixos possui um alto contraste. Em termos da matriz de co-ocorrência, quanto há uma alta concentração de elementos em torno da diagonal principal. Seu cálculo é dado pela equação 5 .

$$
f_{\text {conH }}=\sum_{i=0}^{H g} \sum_{j=0}^{H g}(i-j)^{2} \cdot p(i, j)
$$

3. Homogeneidade: Mede a descrição dos pixels. Quanto mais homogênea a textura, maior seu valor de homogeneidade. Essa medida é apresenta uma correlação inversa com o contraste, pois possui maior concentração na diagonal principal da matriz de co-ocorrência e é dada pela equação 6.

$$
f_{\text {hom }}=\sum_{i=0}^{H g} \sum_{j=0}^{H g} \frac{1}{1+(i-j)^{2}} \cdot p(i, j)
$$

4. Segundo Momento Angular ou Uniformidade: Como já dito em seu nome, este descritor mede a uniformidade de uma imagem. Em termos de matriz de co-ocorrência, ela possui seus valores bem distribuídos ao longo da mesma. Sua fórmula é definida pela equação 7 .

$$
f_{\text {sma }}=\sum_{i=0}^{H g} \sum_{j=0}^{H g} p^{2}(i, j)
$$

5. Correlação: Mede a dependência linear entre dois tons de cinza. Valores altos desta característica indicam uma existência linear entre os pares de tons de cinza. É dada pela equação 8 .

$$
f_{\text {corr }}=\frac{1}{\sigma_{x} \sigma_{y}} \sum_{i=0}^{H g} \sum_{j=0}^{H g}\left(i-\mu_{i}\right)\left(j-\mu_{j}\right) p(i, j)
$$


Onde $\mu_{i}$ e $\mu_{j}$ é dado pelas equações 9 .

$$
\left\{\begin{array}{l}
\mu_{i}=\sum_{i=1}^{H g} i \cdot \sum_{j=1}^{H g} P(i, j) \\
\mu_{i}=\sum_{i=1}^{H g} i \cdot \sum_{j=1}^{H g} P(i, j)
\end{array}\right.
$$

\subsection{Descritores de Textura Baseados Função de Autocorrelação}

Em janeiro de 2011, Abbadeni [1] teve o seu artigo publicado, propondo novos descritores de texturas baseados na função de autocorrelação. Dois de seus descritores foram estudados. Porém, antes de começar a tratar sobre os seus descritores, um estudo em cima da função de autocorrelação foi necessário. Sua fórmula é dada pela equação 10.

$$
\begin{aligned}
f\left(d_{i}, d_{j}\right) & =\frac{1}{\left(n-d_{i}\right)\left(m-d_{j}\right)} \\
& x \sum_{i=0}^{n-d_{i}-1} \sum_{j=0}^{m-d_{j}-1} I(i, j) . I\left(i+d_{i}, j+d_{j}\right)
\end{aligned}
$$

Onde I é a matriz representando os valores de pixels da imagem, $0 \leq d_{i}<n$ e $0 \leq d_{j}<m . d_{i}$ e $d_{j}$ representam o mapeamento das linhas e colunas da função, respectivamente. Diferentemente da Matriz de Co-ocorrência, que realiza suas operações com relação a freqüência entre pixels vizinhos, a Função de Autocorrelação verifica a interação entre regiões. Abbadeni [1] a escolheu por causa de sua periodicidade equivalente e que tem como característica uma lenta diminuição apresentando poucas variações.

1. Rugosidade: De acordo com o Abbadeni [1], a própria função de autocorrelação por si só, já traz informações de rugosidade. A dedução e explicação, disponíveis em seu trabalho, mostram que esta característica depende da quantidade de máximos presentes na função de autocorrelação. É dada pela equação 11.

$$
C s=\frac{1}{\frac{1}{2} x\left(\frac{\sum_{i=0}^{n-1} \sum_{j=0}^{m-1} \operatorname{Max}_{x}(i, j)}{n}+\frac{\sum_{i=0}^{n-1} \sum_{j=0}^{m-1} \operatorname{Max}_{y}(i, j)}{m}\right)} \text { (11) }
$$

Onde $\operatorname{Max}_{x}(i, j)$ e $\operatorname{Max}_{y}(i, j)$ foram obtidos pelo cálculo dos máximos utilizando derivadas parciais em x e y da função de autocorrelação, respectivamente.

2. Ocupação: Possui uma relação inversa com a rugosidade. É calculada a partir do resultado obtido na Rugosidade de uma dada imagem. Seu cálculo é dado pela equação 12 .

$$
f_{b u s y}=1-C_{s}^{1 / a}
$$

Onde $a=4$, segundo Abbadeni [1], dentre os vários valores testados, este foi o que obteve melhores resultados.

\section{Resultados obtidos}

O banco de imagens utilizadas continha um total de 60 imagens, com 512 x 512 pixels em suas dimensões. Um algoritmo para dividir essas imagens em sub-imagens, denominadas de classes, com 128 x 128 pixels de dimensão, ou seja, para cada imagem, 16 classes foram produzidas. $\mathrm{Na}$ fig. 4 mostra uma ilustração de como foi feito o processo de obtenção das classes.

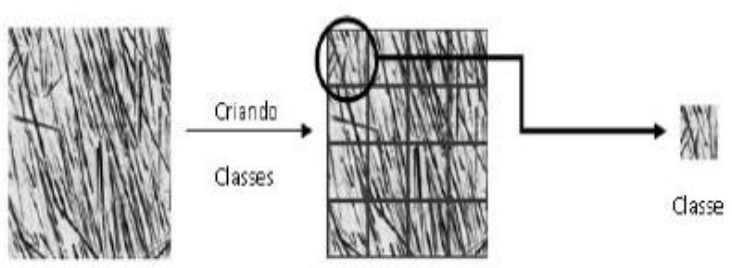

Fig. 4. Imagem ilustrativa de como as classes foram obtidas

Com as classes obtidas, o filtro de Gabor (Afshang [2]) é aplicado para reduzir os ruídos nas classes, e então, as características são alcançadas com os descritores propostos por Abbadeni [1] e Haralick [12]. Com essas características armazenadas, a distância euclidiana é calculada em cima desses valores para uma dada classe. Obtidas as distâncias, ordenam-se todas as distâncias das classes de forma crescente (do mais similar ao menos similar). Com a ordenação, verificam-se quais classes daquelas mais próximas para calcular a curva de recall (Zhang [20]) para uma classe. No eixo $X$, estão as classes ordenadas e no eixo $\mathrm{Y}$ os acertos (classes obtidas de uma mesma imagem matriz).

Realizado todo este processo para uma classe, o mesmo é feito para as outras 959 classes, tendo a curva de recall geral como a média entre as 960 curvas de recall para cada classe. Na fig. 5 , pode ser observado o esquema ilustrativo de todo experimento para obtenção das curvas. 

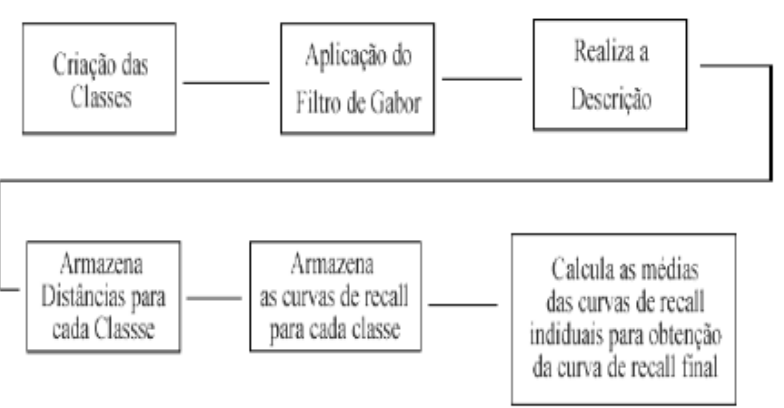

Fig 5. Esquema de como os experimentos foram realizados

Como primeira medida para analisar os resultados obtidos, a descrição do banco de imagem de Brodatz [6], foi realizada utilizando um descritor por vez. É importante frisar que este estudo comparativo serviu para verificar se os novos descritores propostos por Abbadeni [1] seriam relevantes diante dos antigos descritores de Haralick [12]. Realizando a utilização de cada descritor no banco de imagem com 960 classes, o resultado dado por cada curva de recall foi dado pelo que está sendo mostrado na fig. 6 .

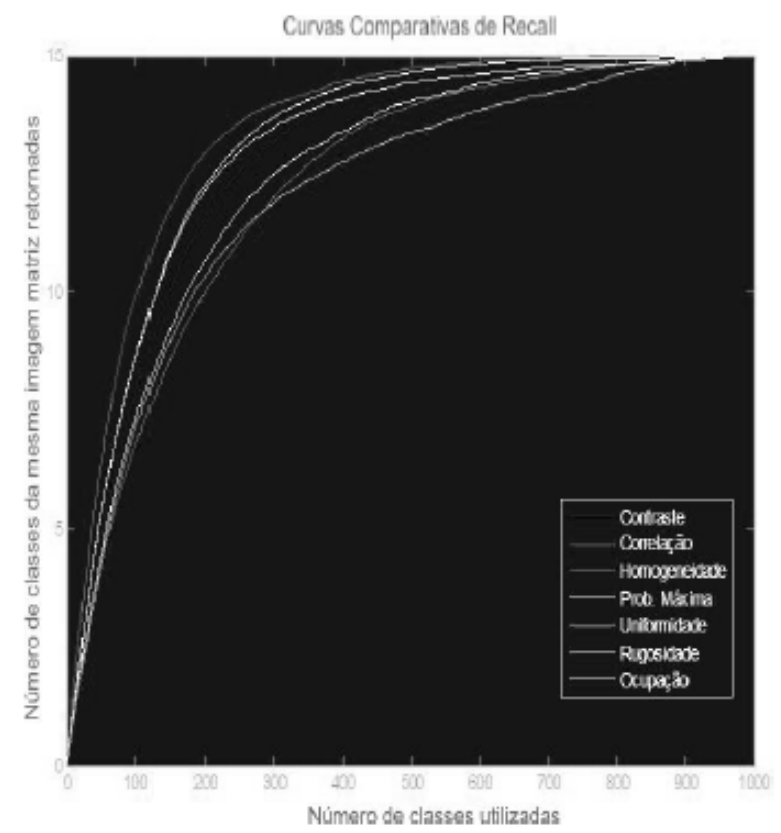

Fig. 6. Comparando curvas de Recall obtidas individualmente para cada descritor de textura

O melhor resultado, que é caracterizado pela curva de maior área, observando as curvas de recall, foi o da descrição de Correlação (em vermelho) de Haralick [12]. As descrições de Ocupação (em beje) e Rugosidade (em dourado), propostas por Abbadeni [1], ficaram em segundo e terceiro lugar respectivamente. Mostrando que a proposta de novos descritores baseada na função de Autocorrelação foi válida para o experimento.
Restava somente, tentar realizar uma descrição que superasse o descritor de Correlação. Então, experimentalmente, foi realizada a combinação das características. Várias combinações foram testadas, mas ficou avaliado que as descrições combinadas com as que ficaram abaixo das três primeiras, não conseguiram superar a curva de recall obtida pela Correlação. Restou combinar a descrição de Correlação com as outras duas melhores, baseadas na função de Autocorrelação. E o resultado foi surpreendente, superando a descrição de Correlação. O que pode ser explicado, já que havendo a combinação entre os descritores de alto índice de informações, mais requisitos podem ser processados e calculados, como pode ser observado na fig. 7 .

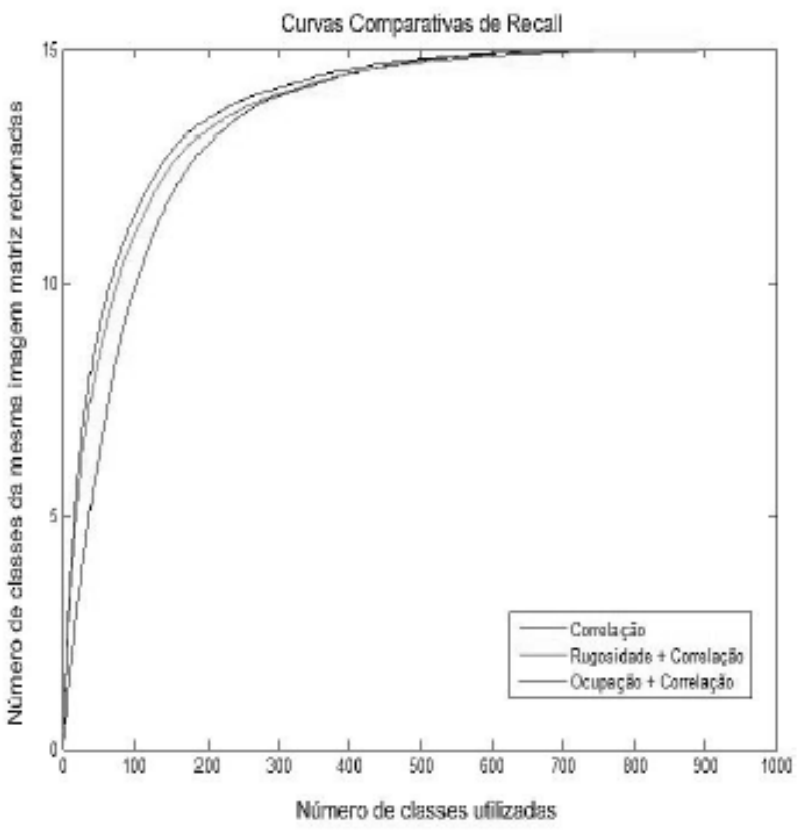

Fig. 7. Curvas de recall combinadas em comparação com a curva do melhor descritor obtido

Portanto, como pode ser observado, combinando os melhores descritores com a Correlação pôde-se obter um resultado melhor do que a Correlação por si só. Para o acerto de 10 classes, por exemplo, a combinação com Ocupação precisou de 66 classes, contra 77 da combinação com Rugosidade e 103 da Correlação.

Com estes dados e análises feitas durante este trabalho, Portanto, como pode ser observado, combinando os melhores descritores com a Correlação pôde-se obter um resultado melhor do que a Correlação por si só. Para o acerto de 10 classes, por exemplo, a combinação com Ocupação precisou de 66 classes, contra 77 da combinação com Rugosidade e 103 da Correlação. 


\section{Conclusão}

Com estes dados e análises feitas durante este trabalho, ficou demonstrado que o melhor descritor para Recuperação de Imagem por imagens foi combinar o descritor de Correlação com o de Ocupação. É de surpreender como descrições propostas nos anos 70, ainda hoje, são utilizadas como fonte de pesquisa, podendo até serem combinados com os novos métodos para a obtenção de melhores resultados.

O estudo de filtros também foi um período de amplo aprendizado, principalmente para o estudo de sinais, assim como suas operações e propriedades. Destaque para o filtro de Gabor, onde sua programação foi relativamente nãotrivial. Felizmente, quando obtido o algoritmo final, a filtragem foi amplamente testada e aproveitada não só para a análise das curvas de recall, como também para realce de imagens. E como previsto, os resultados das curvas melhoraram bastante o com sua propriedade na redução de ruídos.

Para pesquisas futuras, fazer novas combinações com os descritores seja com já propostos ou novos que estejam por vir e a utilização de outros parâmetros para o filtro de Gabor talvez possam melhorar ainda mais os resultados que foram obtidos nesta pesquisa, que certamente foi bem proveitosa e de amplo conhecimento da área de Processamento Digital de Imagens e de CBIR.

\section{Referências}

[1] Abbadeni, N. (2011) "Computacional Perceptual Features for Texture Representation and Retrieval", In: IEEE Transactions on Image Processing.

[2] Afshang, M., Helfroush, M.S. e Zahernia, A., (2009) "Gabor Filter Parameters Optimization for Texture Classification Based on Genetic Algorithm” IEEE, In: Machine Vision, 2009. ICMV.

[3] Aigrain, P., Zhang, H., e Petkovic, D. (1996) "Content-based representation and retrieval of visual media: A review of the state-of-the-art" Multimed. Tools Appl. 3, 3, 179-202.

[4] Asdalogan, Y. e Yu, C. T. (1999) "Techniques and Systems for Image and Video Retrieval" IEEE Transactions, Vol. 11, No. 1. Pp 56-63.

[5] Baraldi, A. e F. Parmiggiani, 1995. "An investigation of the textural characteristics associated with grey level co-occurrence matrix statistical parameters", IEEE Trans. Geosci. Remote Sensing, Vol. 33, 2, pp. 293-304.

[6] Brodatz, P. (1966) "Textures: A Photographic Album for Artists and Designers", In: New York, Dover.

[7] Smith, J. e Chang, S. (1997) "Integrated spatial and feature image query" IEEE Trans. Knowl. Data Eng. 9, 3, 435-447.

[8] Snoek, C. G. M. and Worring, M. (2005) "Multimodal video indexing: A review of the state-ofthe-art" Multimed. Tools Appl. 25, 1, 5-35.

[9] Reaes, F. M. (2006) "Reconhecimento de Faces: Projeto Beholder" Monografia, Universidade de São Paulo.

[10] Datta, R., Joshi, D., Li, J., e Wang, J. (2008) "Image Retrieval: Ideas, Influences, and Trends of the New Age", In: ACM Comput. Surv. 40.

[11] Gonzalez, R. C. e Woods, R. E. (2010) "Digital Image Processing”, Prentice Hall, Inc., Upper Saddle River, NJ, Estados Unidos.

[12] Haralick, R., Shanmugam, K. and Dinstein, I. (1973) "Textural features for image classification” In: IEEE Trans. Syst.

[13] Haralick, R. e Shapiro, L. G. (1992) “Computer and Robot Vision", Vol. 1, Addison-Wesley, Reading, MA, Estados Unidos.

[14] Haralick, R. e Shapiro, L. G. (1993) “Computer and Robot Vision", Vol. 2, Addison-Wesley, Reading, MA, Estados Unidos.

[15] Pedrini, H. e Schwartz, W. R. (2008) “Análise de Imagens Digitais - Princípios, Algoritmos e Aplicações".

[16] Petrakis, E. e Faloutsos, A. (1997) "Similarity searching in medical image databases". IEEE Trans. Knowl. Data Eng. 9, 3, 435-447.

[17] Rui, Y., Huang, T., e Chang, S. (1999) "Image retrieval: Current techniques, promising directions and open issues" J. Visual Commun. Image Represent. $10,1,39-62$.

[18] Smeulders, A. W.,Worring, M., Santinni, S., Gupta, A. e Jain, R. (2000) "Content-based image retrieval at the end of the early years" IEEE Trans. Pattern Anal. Mach. Intell. 22, 12, 1349-1380.

[19] Thomson, W. S. (2001) "Digitally Manipulated Images and Painting" IEEE, Springfield, Missouri, Estados Unidos.

[20] Zhang, J., e Ye, L. (2009) "Ranking Method for Optimizing Precision/Recall of Content-Based 
Image Retrieval" In: Ubiquitous, Autonomic and Trusted Computing, 2009. UIC-ATC '09. Symposia and Workshops on. pp. 356 - 361. 